\title{
DIFERENCIAS SALARIALES DEBIDO AL USO DE COMPUTADORA EN EL TRABAJO EN MÉXICO
}

\section{WAGE DIFFERENCES DUE TO COMPUTER USE AT WORK IN MEXICO}

\section{Aldo Josafat Torres García aldo.jtg@gmail.com \\ UnIVERSIDAD AUTÓNOMA DE}

Chinuahua
Gustavo FélixVerduzco

GUSTAVO.FELIX@UADEC.EDU.MX

UnIVERSIDAD AUtÓNOMA

DE COAHUILA

\section{RESUMEN}

El objetivo del trabajo es contrastar la hipótesis sobre la existencia de una prima salarial por el uso de la computadora en el trabajo. Para la prueba estimamos ecuaciones salariales tipo Mincer. Los resultados indican que el uso de computadora aumenta la disparidad salarial a favor de los usuarios. En un análisis crossection para el año 2012, que controla diversos factores, se estima que los asalariados que utilizan computadora en el trabajo ganan un promedio de 8.2 por ciento más que los no usuarios. Mientras que entre los trabajadores por cuenta propia los usuarios de computadoras ganan, en promedio,19.5 por ciento más.

Palabras clave: cambio tecnológico, computadora, desigualdad salarial, ecuación salarial

Recepción: 8 de agosto de 2018 Aceptación: 30 de octubre de 2018
Revista de Economía - Vol. XXXV - Núm 91

Julio a Diciembre de 2018 - Págs.: 35-63 


\begin{abstract}
The aim of this paper is to contrast the hypothesis about the existence of a wage premium by the computer use at work. For test, we estimate Mincer's wage equations. Results indicate that computer use rise wage disparity in favor of users. In a crossection analysis for 2012, which controls various factors, it is estimated that employees who use a computer at work earn on average 8.2 percent more than non-users. Meanwhile, among self-employed, computer user workers on average earn 19.5 percent more.
\end{abstract}

Keywords: technological change, computer, wage inequality, wage equation.

JEL: O33, J31

\title{
1. INTRODUCCIÓN
}

En los países desarrollados está documentada la existencia de desigualdad salarial vinculada al uso de computadoras en el trabajo; sin embargo, en los países menos desarrollados son escasas las investigaciones en esa dirección. Esto último es lo que motiva el presente estudio, pero también el hecho de que en el país solo 35.8\% de los hogares cuentan con computadora; en países desarrollados es de 75 por ciento; lo que implica que, de existir un premio salarial al uso productivo de computadoras, la desigualdad en las oportunidades para obtener los conocimientos y habilidades necesarias son una fuente adicional que agudiza las desigualdades en la distribución del ingreso.

En general, el análisis de las nuevas tecnologías de la información y comunicación (TIC) y su relación con el mercado laboral es un tema que ha originado investigaciones a partir de varias perspectivas. En particular, se considera que las TIC tienen un impacto positivo sobre el desempeño económico de las empresas y, por lo tanto, al instrumentar esas tecnologías requieren la contratación de trabajadores habilitados en su manejo, en consecuencia se plantea que existe una diferenciación salarial positiva que favorece a ese tipo de trabajadores. 
La revolución tecnológica generada a partir de la creación del microprocesador a principios de la década de 1970 ha impuesto un paradigma tecno económico que ha transformado las interacciones económicas y sociales a nivel mundial (Mochón, González y Calderón, 2014), con tal alcance y profundidad que continúa dimensionándose su trascendencia. En este contexto, las computadoras representan la caracterización más distintiva de la nueva revolución tecnológica.

Estos dispositivos se consideran tecnologías de propósito general (TPG) (Bresnahan y Trajtenberg, 1995; Edquist y Henrekson, 2006; Hagemann, 2008), ya que tienen aplicación genérica en la mayoría de las actividades económicas y han sido incorporadas en los procesos productivos y administrativos para mejorar la eficiencia y generar ventajas competitivas sostenibles (Dutrénit, 2009; Galve y Gargallo, 2004).

Un par de elementos han impulsado favorablemente la diseminación de computadoras durante los últimos años: la reducción de precios y el incremento de actividades basadas en herramientas informáticas, producto de externalidades de red ${ }^{1}$, que se han expandido desde el ámbito científico y laboral hacia el resto de la sociedad. Ambas cuestiones han influido en el mayor uso y aceptación social de esta tecnología, sin embargo, siguen prevaleciendo factores que mantienen una brecha entre quienes forman parte y participan de esta realidad y quienes aún no lo hacen (fenómeno conocido como brecha digital).

Las implicaciones de estas tecnologías en el mercado laboral han sido analizadas por la literatura de la desigualdad salarial y el cambio tecnológico, destacándose dos planteamientos: uno que enfatiza en los factores asociados a la oferta y demanda laboral y el otro que intenta aproximar de manera directa la relación entre el papel de la tecnología y los cambios en la estructura salarial (Autor, Katz y Krueger, 1998; Autor, Levy y Murnane, 2003; Acemoglu, 2002, Card y DiNardo, 2002; Félix y Torres, 2018; Huesca y Ochoa, 2016; Katz y Autor, 1999; Krueger, 1993; Navarro, 2010; Rodríguez y Castro, 2012; Torres y Ochoa, 2018).

\footnotetext{
${ }^{1}$ De acuerdo con Fuentelsaz, Polo y Macías (2003: 56) el término se refiere al incremento de la utilidad obtenida por cada usuario conforme aumenta el total de usuarios del bien o servicio.
} 
El presente trabajo se ubica en el segundo enfoque y se plantea como objetivo contrastar empíricamente la hipótesis sobre la existencia de un premio salarial al uso de computadora en el trabajo. El contraste se realiza considerando por separado a los asalariados de tiempo completo contratados por un patrón y a los trabadores por cuenta propia. Se utilizan datos crossection correspondientes al primer trimestre de 2012, provenientes de la fusión de la Encuesta nacional de ocupación y empleo (ENOE) con el Módulo sobre disponibilidad y uso de tecnologías de la información en los hogares (MODUTIH).

El documento se compone de seis secciones. Después de la introducción se realiza una breve revisión de literatura sobre el uso de la computadora y sus implicaciones en el salario; enseguida se resumen algunos hechos estilizados acerca del uso de esa tecnología en los hogares mexicanos; la cuarta sección describe los datos y la metodología utilizada; en la quinta se analizan los resultados y al final se concluye con algunas reflexiones.

\section{REVISIÓN DE LA LITERATURA: USO DE COMPUTADORA Y SALARIOS}

Si bien los estudios sobre la estructura salarial son tan antiguos como la misma ciencia económica (Katz y Autor, 1999), el drástico aumento de la desigualdad salarial desde finales de la década de 1970 en Estados Unidos y en el Reino Unido generó aún mayor interés sobre el tema (Acemoglu, 2002; Card y DiNardo, 2002; Katz y Autor, 1999; Krueger, 1993).

El incremento de la disparidad salarial se correspondió con el surgimiento de la revolución informática, misma que en el mercado laboral se manifestó a través de la computarización del lugar de trabajo, razón por la cual se ha tratado de explicar el desigual comportamiento de los salarios como consecuencia del cambio tecnológico inducido por el uso de computadoras (Katz y Autor, 1999).

De esta manera, se ha discutido que la incorporación de tecnología informática, en específico el uso de computadoras, representa uno de los principales cambios tecnológicos que han impactado al mercado laboral 
durante las últimas décadas (Autor, Katz y Krueger, 1998; Autor, Levy y Murnane, 2003; Acemoglu, 2002, Caselli, 2014; Card y DiNardo, 2002; Dostie, Jarayaman y Trépanier, 2006; Félix y Torres, 2018; Galve y Garga1lo, 2004; Krueger, 1993; Liu, Tsou y Hammitt, 2004; Torres y Félix, 2017).

En general, se propone que este cambio técnico no es neutral sino sesgado y tiene, por lo menos, dos efectos importantes: la adopción de la nueva tecnología en las empresas incrementa la demanda por trabajadores calificados, disminuyendo la de los no calificados, lo cual, a su vez, ocasiona aumento de los salarios relativos al trabajo calificado, en detrimento del no calificado. Lo descrito es lo que en resumen postula la hipótesis del cambio tecnológico sesgado (HCTS) (Acemoglu, 2002; Autor, Katz y Krueger, 1998; Berman, Bound y Griliches, 1994; Card y DiNardo, 2002; Dunne et al., 2004; Huesca y Ochoa, 2016; Rodríguez y Castro, 2012).

Un elemento fundamental en la dinámica de la HCTS es el estado de complementariedad o sustitución de la tecnología, es decir, si la computadora complementa o sustituye a la actividad humana. Autor, Levy y Murnane (2003) proponen la clasificación entre tareas rutinarias y no rutinarias ("routine tasks" y "nonroutine tasks"), las primeras hacen referencia a un conjunto de actividades que pueden ser realizadas siguiendo reglas explícitas (programables), mientras que las últimas, se refieren a actividades complejas y de reglas poco claras para su ejecución sistemática en un equipo de cómputo (no programables). De ahí que se considere a las tareas rutinarias como actividades características de los trabajadores menos calificados y a las no rutinarias como propias del personal calificado, esto bajo el supuesto de que las actividades no programables requieren de mayor habilidad o formación para ejecutarse (Acemoglu, 2002).

Una vez establecido lo anterior, es posible definir la posibilidad de sustitución o complementariedad del trabajo con la computadora, esto en función del tipo de actividades a realizar, así la tecnología puede sustituir las tareas rutinarias, mientras que en las actividades no rutinarias la computadora tiene efecto complementario (Autor, Levy y Murnane, 2003; Acemoglu, 2002). Al considerar que las actividades rutinarias son frecuentes y poco complejas, en tanto que las no rutinarias menos recurrentes y de 
mayor complejidad, desde la perspectiva de la empresa hay un incentivo para sistematizar actividades de rutina, para de esa forma minimizar los costos por transacciones realizadas dentro de la misma unidad productiva, lo cual lleva a sustituir mano de obra por máquina, mientras que para las tareas no rutinarias se demanda personal ad hoc a la tecnología, es decir, trabajo calificado que sea capaz de realizar tareas complejas mediante el uso de computadora. De esta manera, conforme se adquieren nuevas tecnologías, la demanda relativa de trabajo calificado, así como sus salarios promedio, aumentan (Liu, Tsou y Hammitt, 2004).

Las implicaciones de la HCTS en el comportamiento del empleo y los salarios son por demás relevantes y, puesto que el fenómeno se caracteriza por la incorporación de computadoras, la dinámica del cambio técnico está en buena medida supeditada a la "ley de Moore", la cual establece que la cantidad de transistores de un microchip se duplica cada dos años; traduciéndose en un crecimiento exponencial de la capacidad de cómputo y de la reducción progresiva de su costo (INTEL, 2011). Así, en la medida que la ley de Moore traslade su dinámica a la incorporación de la computadora en los procesos productivos se esperaría, también, una gran dinámica en los efectos del cambio tecnológico sobre la estructura del empleo y los salarios.

Empíricamente, los trabajos seminales de Krueger (1993) y el de DiNardo y Pischke (1997), sentaron las bases para una amplia discusión que se ha ido generado a través de los años sobre la importancia de determinar si las computadoras han tenido implicaciones en los cambios de la estructura salarial, al ser considerada esta tecnología como herramienta auxiliar en la ejecución de determinadas tareas que aumentan la productividad de los trabajadores y, por tanto, se reflejan en una prima salarial para quienes tienen habilidades computacionales.

Krueger (1993) centra la discusión en torno a verificar si los individuos que usan computadora en el trabajo obtienen mayores remuneraciones como resultado de su habilidad informática, comparado con quienes no utilizan computadora; encuentra que, efectivamente, existe un premio para dicha habilidad. Asimismo, manifiesta que el uso de computadoras 
puede explicar, en gran medida, el incremento del rendimiento a la educación ya que, por una parte, los empleados con mayor escolaridad experimentan mayor propensión al uso de estos dispositivos en el trabajo y, por otra, gracias a la gran dispersión y adecuación de la computadora a partir de la década de 1980 en un sinnúmero de actividades. Finalmente, el autor concluye que las computadoras contribuyeron significativamente a los cambios experimentados en la estructura salarial en Estados Unidos.

Por su parte, DiNardo y Pischke plantean la siguiente pregunta: “...ंson las grandes diferencias salariales medidas por el uso de computadoras en el trabajo un verdadero premio a las habilidades computacionales, o simplemente reflejan que los trabajadores con salarios más altos usan las computadoras en sus puestos de trabajo?..." (1997: 291). De esa forma, los autores cuestionan las conclusiones de Krueger (1993) y proponen que las diferencias salariales relacionadas con el uso de otras herramientas de oficina, tales como calculadora, teléfono, bolígrafo/lápiz, e incluso el simple hecho de estar sentado en el trabajo, son casi de la misma magnitud que aquellas atribuidas al uso de equipo de cómputo. DiNardo y Pischke advierten que probablemente existan destrezas no observadas que poco tienen que ver con el uso de la computadora, pero que el mercado laboral también valora.

Tal como se ha reportado, para los países desarrollados está ampliamente documentada la existencia de disparidad salarial vinculada al uso de computadoras. En el caso de países en desarrollo, incluye México, los estudios hasta ahora realizados coinciden con la evidencia encontrada para países desarrollados (Félix y Torres, 2018; Liu, Tsou y Hammitt, 2004; Navarro, 2010; Ng, 2006; Torres y Félix, 2017; Torres y Ochoa, 2018). Solo hemos identificado un trabajo donde la evidencia no es contundente (Oosterbeek y Ponce, 2011), para Ecuador.

Antes, para México buena parte de los trabajos se enfocaban en las implicaciones del cambio tecnológico sobre el mercado laboral y, específicamente, en las disparidades salariales, donde la visualización del cambio tecnológico se realizaba a través de la dinámica en la composición de trabajadores con distintos niveles de calificación representados principalmente por los grados de escolaridad (Castro y Huesca, 2007; Huesca, Castro y 
Rodríguez, 2010; Huesca y Ochoa, 2016; Meza, 2003; Rodríguez y Castro, 2012; Rodríguez, Huesca y Camberos, 2011).

Otra motivación para esta investigación es el hecho de que solamente $35.8 \%$ de los hogares de México cuentan con computadora, mientras que la cifra para países desarrollados es 75\% (INEGI, 2013). Así que, de existir disparidad salarial favorable al uso de la computadora, ésta podría afectar a una gran parte de la población sin acceso.

En resumen, se puede afirmar que la desigualdad salarial en los países desarrollados desde la década de 1980 ha sido explicada por el efecto del cambio tecnológico, caracterizado en buena medida por la intensa incorporación de la computadora y tecnologías afines en los sistemas de producción. En los países menos desarrollados la intensificación del uso individual de computadoras se dio con un importante rezago temporal respecto de los más desarrollados, por lo que la relación entre el uso de tecnología informática y los salarios continúa bajo exploración. La presente investigación pretende contribuir a esta literatura para el caso de México.

\section{EL USO DE LA COMPUTADORA EN LOS HOGARES MEXI- CANOS}

Previo al análisis propuesto, resulta pertinente contextualizar sobre la situación acerca del uso de la computadora en México, en particular, es importante analizar la disponibilidad y uso de computadora en los hogares mexicanos, ya que su diseminación es un indicativo del potencial de las habilidades informáticas de los individuos en el país.

Con base en la información del MODUTIH de 2000 a 2010, en México la posesión de computadoras aumentó más de tres veces: el porcentaje de hogares con computadora pasó de 9.4 a 29.4 por ciento. Para 2008 en Latinoamérica casi la mitad de los países alcanzan una cifra menor a $10 \%$ de tener computadora en el hogar, salvo algunas excepciones como Chile y Costa Rica, donde $25 \%$ de los hogares contaban con computadora (OSILAC, 2007).

De acuerdo con otros estudios realizados por organismos internacionales (OECD, 2001; OSILAC, 2007), contar con bienes de las nuevas tec- 
nologías de la información y comunicación en el hogar está directamente relacionada con el ingreso y el nivel educativo del jefe del hogar. En investigaciones realizadas para México encuentran que el tener computadora y acceso a internet está relacionado positivamente con hogares cuyo jefe tiene estudios universitarios, es mayor de 40 años, con ingresos entre ocho y doce salarios mínimos (Rodríguez, 2005). En Latinoamérica las características sociodemográficas predominantes de los hogares con computadora e internet son: zona urbana, cuatro integrantes, niños en edad escolar, jefe de hogar varón y con edad de entre 35 a 54 años (OSILAC, 2007).

En cuanto al principal uso, para el caso de México el MODUTIH investiga sobre ocho posibilidades. En una primera aproximación y para facilitar el análisis, en este documento se opta por agruparlas en tres categorías: ofimática, educación y esparcimiento. ${ }^{2}$

En lo que sigue, y salvo que se indique lo contrario, toda la información hace referencia al análisis combinado de la Encuesta nacional de ocupación y empleo (ENOE) y del MODUTIH para el primer trimestre de 2012. Así, resulta que $42.2 \%$ de esa población dispone de al menos una computadora en condiciones de uso (el complemento, $57.8 \%$, corresponde a la brecha en cuanto a disponibilidad de computadora). En cuanto al uso, $64.9 \%$ la ocupan principalmente para actividades relacionadas con ofimática; $32 \%$ con fines de esparcimiento y $9.5 \%$ para aplicaciones educativas.

Para asociar el principal uso de acuerdo con la condición de ocupación del usuario se definieron tres categorías: empleados (aquellos que tienen un jefe o superior), trabajadores por cuenta propia y desocupados. ${ }^{3}$ La distribución de la población con base en esas categorías es del 39, 15.1

\footnotetext{
${ }^{2}$ En ofimática se agruparon las siguientes aplicaciones: manejadores de bases de datos, lenguajes de programación, hojas de cálculo, programas de administración, y editores de imagen/gráficas. En el uso para educación se consideran los programas para apoyar la enseñanza/aprendizaje. En la categoría de esparcimiento se incluyen los programas de juegos y de comunicación.

${ }^{3}$ Este concepto se refiere al de desempleo abierto, considerado en la ENOE como la parte de la población económicamente activa disponible para trabajar, que está sin trabajo y en búsqueda de trabajo.
} 
y 45.9 por ciento, respectivamente. Por su parte, la utilización de computadora se distribuye en $47,26.2$ y 56 por ciento.

La gráfica 1 muestra que 53\% de los empleados no disponen de computadora en el hogar; el complementario $47 \%$ si cuenta con ella, $28.3 \%$ la utiliza principalmente en aplicaciones ofimáticas, $4.7 \%$ en aplicaciones relacionadas con la educación y $14.4 \%$ en las de esparcimiento.

La gran mayoría de los que trabajan por su cuenta (73.8\%) no disponen de computadora; $26.2 \%$ que sí cuenta con ella se distribuye en: $16.2 \%$ para uso principal de ofimática, $1.5 \%$ en programas de educación y $8.5 \%$ en esparcimiento.

De los desocupados 44\% no tienen computadora en el hogar, el restante $56 \%$ que si la posee se compone de: $20.8 \%$ la usa principalmente en programas de ofimática, $7.2 \%$ en programas relacionados con la educación y $28 \%$ en programas clasificados como de esparcimiento.

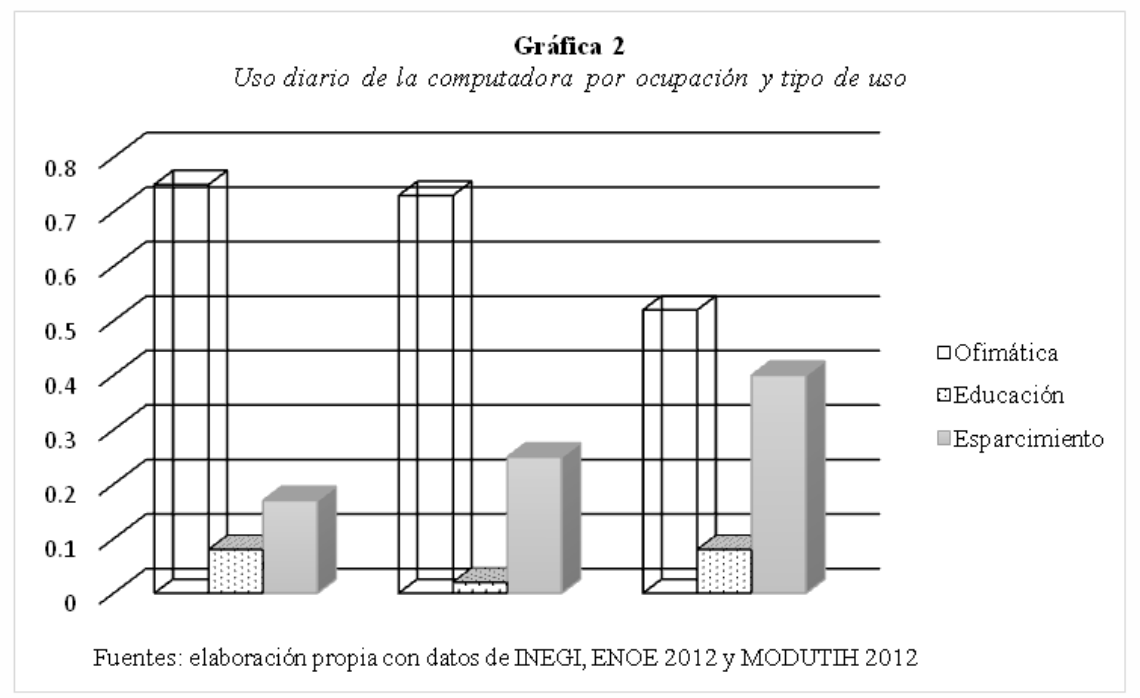

Posteriormente, los individuos fueron clasificados de acuerdo con su ingreso, medido en términos del salario mínimo. Independientemente del nivel de ingreso, 49\% dispone de computadora en el hogar (ráfica 3). Ahora bien, en los grupos que declararon tener ingresos, el porcentaje de dispo- 
nibilidad crece con el nivel del mismo: así, de los que ganan más de seis salarios mínimos $80.5 \%$ dispone de computadora; en el grupo de tres a seis salarios mínimos el porcentaje disminuye a 57.9; en los de 2 a 3 salarios es $40 \%$; en los de uno a dos salarios $27.4 \%$ y $22 \%$ en los que ganan menos de un salario mínimo. Claramente se observa que, en efecto, hay una correlación positiva entre el ingreso y la disponibilidad de computadora, lo cual es razonable debido al mayor poder de compra. Aunque debe destacarse que casi la mitad de los individuos (48.9\%) que al momento de la encuesta declararon no tener ingresos también disponen de computadora.

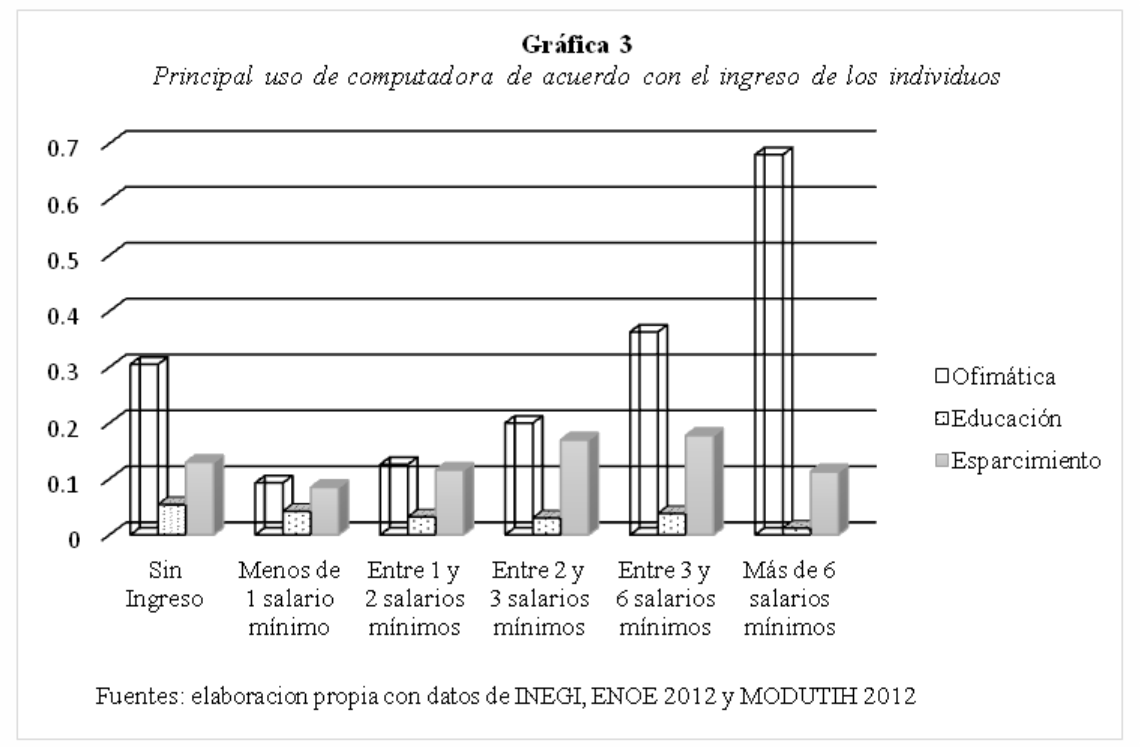

El análisis hasta aquí descrito verifica que en diez años se ha reducido la brecha en la disponibilidad de computadora en los hogares mexicanos: el porcentaje de hogares con carencia de computadora disminuyó de 90.6 en el año 2000 a 70.6 en 2010. Esta reducción, aunque significativa, no es comparable con la observada en países desarrollados como Estados Unidos, donde en los mismos años la falta de equipo de cómputo en los hogares se redujo de 49 a 23.3 por ciento, para el Reino Unido entre el año 2000 y 2009 la cifra disminuyó de 62 a 18.8 por ciento; en ese mismo pe- 
ríodo Suiza y Finlandia redujeron la carencia de computadora en el hogar de 42.3 a 19 y de 53 a 19.9 por ciento, respectivamente (OECD, 2012).

Para el caso de México, destaca que los individuos que no están laborando son quienes en mayor porcentaje disponen de computadora en el hogar, lo cual es lógico puesto que en este grupo se ubican quienes continúan estudiando. En los individuos que trabajan es mayor el porcentaje de disponibilidad en el grupo de empleados con patrón, esto en comparación de quienes laboran por cuenta propia.

\section{DATOS Y METODOLOGÍA}

La información utilizada para el análisis empírico proviene de la combinación de la ENOE y del MODUTIH correspondientes al primer trimestre de 2012. Ambas encuestas son representativas a nivel nacional, la primera contiene información sobre características sociodemográficas y laborales de los individuos y permite ser asociada con la segunda, que proporciona información sobre el uso y disponibilidad de computadora. Para el análisis empírico nos interesa la información sobre individuos que trabajan de tiempo completo. La base de datos combinada arroja un total de 6572 individuos de 15 años y más; 4949 son asalariados de tiempo completo empleados por un patrón y 1623 son trabajadores de tiempo completo empleados por cuenta propia.

La estrategia de análisis empírico consistió en realizar primero una regresión por mínimos cuadrados ordinarios (MCO), se considera como variable dependiente al logaritmo natural del salario por hora y como independientes aquellas referentes al uso de equipo de cómputo y una serie de variables que al mismo tiempo sirven para controlar la heterogeneidad no observable. Estas reflejan otras características de los individuos, de las ocupaciones, del empleo y otras características regionales que también influyen en la determinación de los salarios. Al considerar que la determinación del ingreso laboral es distinta este modelo se aplica por separado, por un lado, a los asalariados de tiempo completo empleados por patrón y, por otro, a los trabajadores de tiempo completo empleados por cuenta propia. 
Para el caso de los trabajadores empleados por patrón se estima un segundo modelo que controla el sesgo por selección utilizando el método de Heckman (1979), esto para considerar el hecho de que los trabajadores usurarios de computadora cuentan también con otro tipo de habilidades reconocidas y premiadas por las empresas. En todas las estimaciones se utiliza la técnica de bootstrap para obtener errores estándar robustos.

Así entonces, para los trabajadores empleados por un patrón la ecuación salarial de Mincer (1974) es:

$$
\ln w_{i}=\beta_{0}+\delta_{1} \text { dpctrabajar }_{i}+x_{i} \beta+u_{i}
$$

Donde $\ln w_{i}$ es el logaritmo natural del salario por hora. Las variables explicativas estan representadas por dpctrabajar ${ }_{i}$, variable binaria correspondiente al uso de computadora en el trabajo, el vector $x_{i}$ incluye otras características individuales del empleo y regionales, que también influyen sobre los salarios. ${ }^{4}$ El término $\ln \left(w_{i}\right)$ es la perturbación aleatoria que se distribuye bajo los supuestos usuales.

Se supone que, si las empresas buscan contratar trabajadores con habilidades, estas preferirían a quienes las poseen, por lo que la selección de trabajadores a estudiar debería limitarse solo a aquellos que cuentan con habilidades en el uso de computadora. Sin embargo, la estimación de (1) bajo esas condiciones, de acuerdo con Heckman (1979), podría representar un sesgo de selección y el coeficiente asociado a la variable de interés estaría sobreestimado. Para corregir el sesgo, la solución de Heckman consiste en estimar dos ecuaciones, una de ellas es la ecuación (1) y la otra es una función que determina la probabilidad de que el trabajador cuente con dichas habilidades (ecuación de selección). Si las perturbaciones aleatorias

\footnotetext{
${ }^{4}$ Nueve variables dummy de rama, una continua de horas trabajadas, tres dummy sobre condiciones de ocupación para individuos que laboraron menos de 35 horas, ocupados que trabajan 35 horas o más y ganan hasta un salario mínimo, así como ocupados que trabajan más de 48 horas con ingresos de más de 1 y hasta 2 salarios mínimos, cuatro variables dummy referentes al tamaño del establecimiento, nueve dummy de categorías de ocupación y treinta y siete variables dummy que hacen referencia a las ciudades autorepresentadas de la ENOE.
} 
de ambas ecuaciones están correlacionadas, entonces existe sesgo de selección, y es necesaria la corrección.

En el caso de trabajadores por cuenta propia se supone que su producto es más competitivo si se auxilian con el uso de computadora, independientemente del lugar donde lo hagan, por ese motivo se postula que obtienen un premio al ingreso cuando poseen habilidades. Adicionalmente, al ser trabajo por cuenta propia, no existe un proceso de selección, tampoco un lugar fijo de trabajo, por lo que la variable explicativa referente a la habilidad considera únicamente su capacidad para utilizar computadora, por lo tanto la ecuación correspondiente es:

$$
\operatorname{lnw}_{i}=\beta_{0}+\gamma_{1} u \operatorname{sapc}_{i}+x_{i} \beta+v_{i}
$$

El cuadro 1 ofrece una descripción general de las variables utilizadas. Se observa que para los dos tipos de trabajadores, asalariados y por cuenta propia, los usuarios de computadora en el trabajo tienen una media de salario por hora superior a quienes no la utilizan; no obstante, la escolaridad promedio también es mayor para ambos subconjuntos de trabajadores, por ese motivo, el mero análisis descriptivo no permite determinar en qué medida cada uno de esos dos factores influyen sobre las diferencias salariales. También coincide que la experiencia potencial (años transcurridos después del último grado de estudios) es menor en los usuarios de computadora, lo que indica su uso mayoritario por personas jóvenes. El porcentaje de trabajadores por cuenta propia que utiliza computadora (17.7\%) está sensiblemente por debajo de los asalariados que la utilizan para trabajar (27.7 por ciento). En ambos grupos de trabajadores, la mujer es la que mayoritariamente utiliza computadora: $34.4 \%$ de mujeres asalariadas utilizan computadora en el trabajo, esto frente al $24.1 \%$ de los hombres. Por su parte, $19.6 \%$ de mujeres que trabajan por su cuenta son usuarias de computadora y de los hombres 16.6 por ciento. 
Cuadro 1. Trabajadores que utilizan computadora, 2012

\begin{tabular}{|l|cc|cc|}
\hline \multirow{2}{*}{ Variables } & \multicolumn{2}{|c|}{$\begin{array}{c}\text { Para trabajar: asalariados con } \\
\text { patrón }\end{array}$} & \multicolumn{2}{c|}{$\begin{array}{c}\text { Trabajadores por su } \\
\text { cuenta }\end{array}$} \\
\cline { 2 - 5 } & No & $S i$ & No & $S i$ \\
\hline Salario por hora (media pesos) & 21.1 & 34.0 & 17.2 & 40.7 \\
Ln Salario por hora (media) & 2.9 & 3.5 & 2.6 & 3.4 \\
Escolaridad (media años) & 8.0 & 13.6 & 6.0 & 12.2 \\
Experiencia potencial (media & & & & \\
años) & 20.7 & 15.8 & 34.8 & 20.1 \\
Horas trabajadas (media & & & & 37.8 \\
semanal) & 42.5 & 41.9 & 1,816 & 390 \\
Número de personas & 4,244 & 1,628 & 82.3 & 17.7 \\
Personas (\%) & 72.3 & 27.7 & 80.4 & 19.6 \\
Mujeres (\%) & 65.7 & 34.4 & 83.4 & 16.6 \\
Hombres (\%) & 76.0 & 24.1 & 76.8 & 23.2 \\
Solteros (\%) & 71.0 & 29.1 & 84.2 & 15.8 \\
Casados (\%) & 73.2 & 26.8 & & \\
\hline
\end{tabular}

Fuente: elaboración propia con datos de INEGI, ENOE 2012 y MODUTIH 2012.

\section{RESULTADOS}

El cuadro 2 presenta los resultados de regresión tanto para los asalariados de tiempo completo empleados por un patrón como para los trabajadores por cuenta propia. Con la excepción de la variable relacionada con el uso de computadora, el cuadro incluye las variables que tradicionalmente se utilizan en los modelos tipo Mincer (1974), el resto de las variables de control que también influyen en el salario se reportan en los cuadros anexados al final del artículo.

Los resultados estimados de la ecuación (1) para los asalariados empleados por patrón se presentan en las columnas 1 a 4 . La columna (1) contiene los coeficientes estimados del modelo MCO y la (3) los correspondientes al modelo con corrección del sesgo por selección. La diferencia porcentual del salario entre usuarios y no usuarios se $\left[\exp \left(\hat{\delta}_{1}\right)-1\right] \times 100$ én los trabajadores por cuenta propia se utiliza $\hat{\gamma}_{1}$ ). 
Las columnas (1) y (3) muestran que, si no se controla el sesgo por selección, los coeficientes sobre diferencias salariales atribuidas al uso de computadora en el trabajo se sobreestiman por poco más del doble, por lo tanto, los resultados a analizar corresponden al modelo Heckman. De igual forma, la prueba de razón de verosimilitud rechaza la hipótesis nula de que la ecuación salarial y la probabilidad de utilizar computadora son independientes, lo que confirma como más adecuado para los trabajadores asalariados el modelo que corrige el sesgo de selección.

El coeficiente con valor 0.079 asociado a la variable dpctrabajar, que indica la utilización de computadora en el trabajo, significa que los usuarios obtienen en promedio un premio salarial de $8.2 \%$ más que los no usuarios.

El resto de los coeficientes tienen valores congruentes a los encontrados en otras investigaciones que estiman ecuaciones salariales tipo Mincer. Así, puede verse que hay también un premio a la escolaridad (esco) equivalente a $9.3 \%$ promedio por año; que los hombres (hombre) ganan en promedio $11.2 \%$ más que las mujeres; que por cada año de experiencia potencial (experi y experi^2/100] ganan $2.3 \%$ más de salario. La variable que indica el estado civil (edocivil) no resultó estadísticamente significativa en la explicación de los salarios. En definitiva, los resultados indican la existencia de un premio al capital humano, dentro del cual la habilidad para utilizar computadora en el trabajo representa casi $10 \%$ para los asalariados de tiempo completo.

Respecto a los empleados por cuenta propia que tienen habilidades para utilizar computadora, en este caso no hay un problema de selección, por lo que los resultados del modelo estimado por MCO son válidos, mismos que se presentan en las columnas 5 y 6 del cuadro 2. Debido a que los trabajadores por cuenta propia pueden auxiliarse en el uso de computadora para mejorar la eficiencia en la provisión de un producto o servicio, la variable de control, en este caso, indica la habilidad y utilización de computadora independientemente del lugar donde se utiliza, que puede ser el hogar, el lugar de trabajo u otro lugar público. 
Los resultados de la columna (5) indican que los trabajadores por cuenta propia que utilizan computadora obtienen, en promedio, un premio al ingreso $19.5 \%$ más que los no usuarios. Así mismo, que el rendimiento asociado a cada año de escolaridad (2.3\%) es sensiblemente más bajo que los obtenidos por los trabajadores asalariados, lo que puede entenderse si se considera que, contrario a lo que ocurre con los asalariados, en la retribución por la provisión de un producto o servicio por cuenta propia se valora más la eficiencia y menos la credencialización atribuible a la escolaridad. Otro resultado que llama la atención es la gran disparidad de ingresos atribuible a la diferencia por género: las estimaciones indican que cuando los trabajadores por cuenta propia son hombres y se mantienen los demás factores constantes, ganan en promedio $44.5 \%$ más que las mujeres. Los resultados relacionados con la experiencia potencial son menores, aunque muy similares a los de los trabajadores asalariados ( 2 contra $2.3 \%$ de estos últimos).

Cuadro 2. Resultados econométricos de las ecuaciones salariales, 2012 Variable dependiente: logaritmo natural de salario por hora

\begin{tabular}{|c|c|c|c|c|c|c|}
\hline \multirow{2}{*}{ Variables } & \multicolumn{4}{|c|}{ Asalariados empleados por patrón } & \multicolumn{2}{|c|}{$\begin{array}{c}\text { Trabajadores por cuenta } \\
\text { propia }\end{array}$} \\
\hline & $\begin{array}{c}M C O \\
(1)\end{array}$ & $\begin{array}{l}\text { Bootstrap } \\
\text { E.S. }\end{array}$ & $\begin{array}{l}\text { Heckman } \\
\text { (3) }\end{array}$ & $\begin{array}{c}\text { Bootstrap } \\
\text { E.S. }\end{array}$ & $\mathrm{MCO}(5)$ & Bootstrap E.S. \\
\hline $\begin{array}{l}\text { dpctrabajar } \\
\text { usapc }\end{array}$ & 0.185 & 0.021 & 0.079 & 0.026 & 0.178 & 0.079 \\
\hline esco & 0.031 & 0.002 & 0.089 & 0.006 & 0.023 & 0.006 \\
\hline hombre & 0.127 & 0.012 & 0.106 & 0.022 & 0.368 & 0.046 \\
\hline edocivil ++ & 0.036 & 0.015 & 0.043 & 0.021 & -0.016 & 0.041 \\
\hline experi & 0.014 & 0.002 & 0.023 & 0.003 & 0.019 & 0.005 \\
\hline experi^ $2 / 100$ & -0.017 & 0.003 & -0.031 & 0.009 & -0.0003 & 0.0001 \\
\hline $\mathrm{N}$ & 4949 & & 4949 & & 1623 & \\
\hline Censuradas & & & 2852 & & & \\
\hline No censuradas & 0.561 & & 2097 & & & \\
\hline $\mathrm{R} 2$ ajustado & 7 & & & & 0.5471 & \\
\hline $\begin{array}{l}\text { rho } \\
\text { p-value LR }\end{array}$ & & & 0.69 & & & \\
\hline test & & & 0.0001 & & & \\
\hline
\end{tabular}

No se reportan las variables de control. El resto de los resultados puede consultarse en el anexo. ++ única variable que resultó no significativa. El resto de las variables son significativas con p-value menor a 0.05. Fuente: Estimaciones propias con datos de INEGI, ENOE 2012 y MODUTIH 2012, primer trimestre. 


\section{CONCLUSIONES}

Con datos de 2012 se estimaron ecuaciones salariales tipo Mincer (1974) correspondientes a trabajadores asalariados y por cuenta propia; los resultados sobre los rendimientos a la escolaridad, la experiencia y género de los trabajadores son congruentes con los hallazgos en otros estudios.

La innovación en esta investigación fue determinar las diferencias salariales que obtienen los trabajadores como un premio a la habilidad por el uso productivo de la computadora. Se encontró que los trabajadores asalariados obtienen un premio salarial promedio de 8.2 por ciento. Por su parte, el premio para los empleados por cuenta propia es sensiblemente superior, ubicándose en un promedio de 19.5 por ciento.

Los resultados reportados para México en el año 2012 no están muy alejados de los encontrados para países desarrollados: entre 10 y $15 \%$ reportados por Krueguer (1993) para Estados Unidos; Entorf y Kramarz (1997) estiman premios entre 6 y 10\% en el caso de Francia; Reilly (1995) para Canadá encontró que los usuarios de computadora en el trabajo ganaban 13.5\% más que los no usuarios en 1979; Arabsheibani, Emami y Marin (2004) para el Reino Unido consideran premios salariales de 23 y 20\% en 1985 y 1990; Miller y Mulvey (1997) estiman para Australia, premios de 10 a 15\% en 1993; Liu, Tsou y Hammitt (2004), con datos de 1999 para Taiwán encuentran que los individuos usuarios de computadora en el trabajo ganan $14 \%$ más que los no usuarios. Las diferencias entre países se deben, principalmente, a las condiciones específicas respecto de la demanda y oferta de trabajadores con habilidades en el uso productivo de la computadora, lo cual es cambiante en el tiempo.

Para el caso de México, la combinación de resultados: mayor premio salarial al uso de computadora y menor rendimiento a la educación y a la experiencia potencial de los trabajadores por cuenta propia, en comparación con los trabajadores asalariados, son congruentes con la posibilidad de que la retribución al trabajo por cuenta propia esté basada más en la calidad y eficiencia del producto o servicio, y menos en la credencialización y el escalafón por antigüedad aplicado comúnmente en las empresas. 
La existencia de un premio a la habilidad por el uso de computadora implica un elemento adicional que se agrega a la reproducción de otro tipo de desigualdades en el país. Así, la desigualdad en las oportunidades para acceder a niveles educativos de calidad se suma a la desigualdad para tener la formación adecuada y adquirir habilidades en el uso productivo de la computadora, lo que finalmente incrementa aún más las desigualdades salariales.

Se vio que, a pesar de uso de computadoras en México se ha incrementado en los últimos años, aún existe una brecha significativa en la tenencia de estos equipos en los hogares mexicanos, sobre todo si se le compara con los hogares de países desarrollados. Ese resultado se traduce al mercado laboral, dónde únicamente el 27.7 por ciento de asalariados utilizan computadora en el trabajo y sólo el 17.7 por ciento de trabajadores por cuenta propia están habilitados en el uso de computadora.

\section{REFERENCIAS}

Acemoglu, D. 2002. "Technical change, inequality, and the labor market", Journal of Economic Literature, 40(1): 7-72.

Arabsheibani, R., J. M. Emami y A. Marin. 2004. "The impact of computer use on earnings in the UK", The Scottish Journal of Political Economy, 51: 82-94.

Autor, D., F. Levy y R. Murnane. 2003. "The skill content of recent technological change: An empirical exploration", Quarterly Journal of Economics, 118(4): 1279-1333.

Autor, D., L. Katz y A. Krueger. 1998. "Computing inequality: Have computers changed the labor market?", Quarterly Journal of Economics, 113(4): 1169-1213.

Berman, E., J. Bound y Z. Griliches. 1994. "Changes in the demand for skilled labor within US manufacturing: evidence from the annual survey 
of manufacturers", The Quarterly Journal of Economics, 109(1): 367-397. Bresnahan, T. y M. Trajtenberg. 1995. "General purpose technologies "engines of growth?”, Journal of econometrics, 65: 83-108.

Card, D. y J. Di Nardo. 2002. "Skill-biased technological change and rising wage inequality: Some problems and puzzles", Journal of Labor Economic, 20(4): 733-783.

Caselli, M. 2014. "Trade, skill-biased technical change and wages in Mexican manufacturing”, Applied Economics, 46(3): 336-348.

Castro, D. y L. Huesca. 2007. "Desigualdad salarial en México: una revisión”, Papeles de Población, 54: 225-264.

DiNardo, J. y J. S. Pischke. 1997. "The returns to computer use revisited: Have pencils changed the wage structure too?" The Quarterly Journal of Economics, 112(1): 291-303.

Dostie, B., R. Jayaraman y M. Trépanier. 2006. "The returns to computer use revisited, again”, Institute for the Study of Labor (IZA), DP núm. 2080.

Dunne, T., L. Foster, J. Haltiwanger y K. Troske. 2004. "Wage and productivity dispersion in US manufacturing: The role of computer investment", Journal of Labor Economics, 22(2): 397-429.

Dutrénit, G. 2009. Sistemas regionales de innovación: un espacio para el desarrollo de las pymes. El caso de maquinados industriales, TextualUAM Press, México.

Edquist, H. y M. Henrekson. 2006. “Technological breakthroughs and productivity growth", SSE/EFI Working Paper Series in Economics and Finance, núm. 562. 
ENOE. 2012. Encuesta Nacional de Ocupación y Empleo, base de datos, disponible en: www.beta.inegi.org.mx/proyectos/enchogares/regulares/enoe/

Entorf, H. y F. Kramarz . 1997. "Does unmeasured ability explain the higher wages of new technology workers?" European Economic Review, 41: 1489-1509.

Félix-Verduzco, G. y A. Torres García. 2018. "Prima salarial al uso de computadora en el trabajo. Evidencia de microdatos para México", El Trimestre Económico, 85(337): 137-168. DOI: http://dx.doi.org/10.20430/ete. v85i337.662

Fuentelsaz, L., Y. Polo y J.P. Macías. 2003. "Economía digital y estrategia empresarial: un análisis desde la dirección estratégica", Revista de Empresa, 5: 54-69.

Galve, C. y A. Gargallo. 2004. "Impacto de las tecnologías de la información en la productividad de las empresas españolas", Documento de Trabajo 2004-05, Universidad de Zaragoza.

Hagemann, H. 2008. "Consequences of the new information and communication technologies for growth, productivity and employment", Competitiveness Review: An International Business Journal, 18(1/2): 57-69.

Heckman, J. 1979. “Sample selection bias as a specification error", Econometrica, 47(1): 153-161.

Huesca, L., D. Castro y R.E. Rodríguez. 2010. “Cambio tecnológico y sus efectos en el mercado de trabajo: una revisión analítica”, Economía, Sociedad y Territorio, 10(34): 749-779.

Huesca, L., y G. Ochoa. 2016. Desigualdad salarial y cambio tecnológico en la frontera norte de México, Problemas del Desarrollo, 47(187): 165-188. Instituto Nacional de Estadística y Geografía (INEGI). 2013. Estadísticas 
sobre disponibilidad y uso de las tecnologías de información y comunicaciones en los hogares..México: INEGI.

INTEL. 2011. “1971-2011: 40 años del microprocesador”, recuperado el 26/11/2016: www.intel.com/content/www/us/en/history/history-intelchips-timeline-poster.html

Katz, L. y D. Autor. 1999. "Changes in the wage structure and earnings inequality", en O. Ashenfelter y D. Card, Handbook of Labor Economics, vol. 3A, North-Holland, pp. 1463-1555.

Krueger, A. 1993. "How computers have changed the wage structure: Evidence from Microdata, 1984-1989”, Quarterly Journal of Economics, 108(1): 33-61.

Liu, J.T., M.W. Tsou y J.K. Hammitt. 2004. "Computer use and wages: Evidence from Taiwan", Economics Letters, 82(1): 43-51.

Meza, L. 2003. "Apertura comercial y cambio tecnológico efectos en el mercado laboral mexicano”, El Trimestre Económico, 70 (3): 456-505. Miller, P. y C. Mulvey. 1997. "Computer skills and wages" Australian Economic Papers, 36: 106-113.

Mincer, J. 1974. Schooling, Experience, and Earnings, NBER and Columbia University Press.

Mochón, F., J.C. González y J. Calderón. 2014. Economía digital curso Mooc, editorial Alfaomega.

MODUTIH. 2012. Módulo sobre disponibilidad y uso de tecnologías de la información y comunicación en los hogares, base de datos, disponible en:www.beta.inegi.org.mx/proyectos/enchogares/modulos/modutih/2012/ Navarro, L. 2010. The impact of internet use on individual earnings in Latin America, INESAD, núm. 11/2010. 
Ng, Y.C. 2006. "Levels of computer self-efficacy, computer use and earnings in China", Economics Letters, 90(3): 427-432.

OECD. 2001. Understanding the digital divide, OECD, París.

OECD. 2012. Factbook 2011-2012: Economic, environmental and social statistics, Paris. http://www.iri.edu.ar/publicaciones_iri/anuario/cd_anuario_2012/cooperacion/108.pdf

Oosterbeek, H. y J. Ponce. 2011. "The impact of computer use on wages in a developing country: Evidence from Ecuador", Labour Economics, 18: 434-440.

OSILAC .2007. "Patrones de penetración de los bienes y servicios TIC en los hogares de América Latina y el Caribe. Análisis basado en datos de los censos nacionales de población y vivienda de la ronda del 2000", CEPALNaciones Unidas.

Reilly, K.T. 1995. "Human capital and information: The employer sizewage effect", The Journal of Human Resources, 30(1): 1-18.

Rodríguez, A. 2005. Tecnologías de la información y brecha digital en México 2001-2005, Universidad Nacional Autónoma de México.

Rodríguez, R. y D. Castro. 2012. "Efectos del cambio tecnológico en los mercados de trabajo regionales en México", Estudios fronterizos, nueva época,13(26): 141-174.

Rodríguez, R., L. Huesca y M. Camberos. 2011. "Mercado laboral, inequidad salarial y cambio tecnológico regional”, Frontera Norte, 23(45): 7-33. Torres, A. y G. Félix-Verduzco. 2017. "Rendimiento al uso de PC en México: un análisis comparativo por sector de actividad", en V. Avalos et al. (comps.), Compendio de resultados de investigación aplicada en la ingeniería, Universidad Autónoma de Coahuila, pp. 139-155. 
Torres, A. y G. Ochoa. 2018. "Desigualdad salarial asociada al uso de TIC en México: un análisis por ocupaciones", Cuadernos de Economía, 37(74): 353-389.

ANEXO

Cuadro A1. Complemento a los resultados de regresión del modelo MCO aplicado a empleados asalariados.

\begin{tabular}{|c|c|c|c|c|}
\hline Var dep: ling $x \mathrm{hrr}$ & Coheficiente & Bootstrapp E.S & $Z$ & $p$-value \\
\hline \multicolumn{5}{|c|}{ En este lugar van las variables incluidas en el cuadro 2 del articulo } \\
\hline ocmenos $35 \mathrm{~h}$ & 0.24 & 0.11 & 2.26 & 0.024 \\
\hline ocmsa $35 \mathrm{hsm}$ & -1.08 & 0.05 & -23.08 & 0 \\
\hline ocms: $48 \mathrm{~h} 2 \mathrm{sm}$ & -0.43 & 0.02 & -22.15 & 0 \\
\hline ded_1 & 0.15 & 0.03 & 4.53 & 0 \\
\hline dod_2 & 0.20 & 0.04 & 4.44 & 0 \\
\hline dod_3 & 0.26 & 0.04 & 7.09 & 0 \\
\hline dod_4 & 0.15 & 0.04 & 3.5 & 0 \\
\hline ded_5 & 0.20 & 0.05 & 3.83 & 0 \\
\hline dod_ 7 & 0.13 & 0.06 & 2.27 & 0.023 \\
\hline dod_8 & 0.06 & 0.07 & 0.89 & 0.376 \\
\hline dod_9 & 0.12 & 0.07 & 1.72 & 0.085 \\
\hline dod_10 & 0.11 & 0.06 & 1.83 & 0.067 \\
\hline ded_12 & 0.24 & 0.08 & 2.84 & 0.005 \\
\hline dod_13 & -0.08 & 0.05 & -1.84 & 0.066 \\
\hline dod_14 & 0.10 & 0.04 & 2.39 & 0.017 \\
\hline dod_15 & 0.15 & 0.09 & 1.63 & 0.104 \\
\hline ded_16 & 0.01 & 0.05 & 0.24 & 0.809 \\
\hline ded_17 & 0.09 & 0.15 & 0.63 & 0.526 \\
\hline dod_18 & 0.26 & 0.07 & 4.01 & 0 \\
\hline ded_19 & -0.07 & 0.05 & -1.51 & 0.132 \\
\hline ded_21 & 0.19 & 0.05 & 3.65 & 0 \\
\hline ded_24 & 0.24 & 0.08 & 3 & 0.003 \\
\hline ded_25 & 0.28 & 0.06 & 4.36 & 0 \\
\hline dod_26 & -0.14 & 0.07 & -2.11 & 0.034 \\
\hline dod_27 & 0.27 & 0.05 & 4.97 & 0 \\
\hline ded_28 & 0.08 & 0.07 & 1.15 & 0.252 \\
\hline dod_29 & 0.05 & 0.09 & 0.55 & 0.581 \\
\hline dod_31 & 0.03 & 0.09 & 0.33 & 0.745 \\
\hline ded_32 & 0.22 & 0.08 & 2.75 & 0.006 \\
\hline dod_33 & 0.30 & 0.08 & 3.89 & 0 \\
\hline dod_36 & 0.20 & 0.04 & 4.59 & 0 \\
\hline dod_39 & 0.00 & 0.07 & 0 & 1 \\
\hline dod_40 & 0.27 & 0.07 & 4.09 & 0 \\
\hline dod_41 & 0.18 & 0.05 & 3.37 & 0.001 \\
\hline
\end{tabular}




\begin{tabular}{lrrrr|} 
dcd_81 & 0.14 & 0.02 & 6.37 & 0 \\
dcd_82 & 0.02 & 0.03 & 0.73 & 0.464 \\
dcd_83 & -0.02 & 0.02 & -0.69 & 0.492 \\
dcd_84 & 0.01 & 0.03 & 0.54 & 0.592 \\
dcd 85 & -0.02 & 0.03 & -0.82 & 0.413 \\
dram2 & 0.34 & 0.07 & 4.82 & 0 \\
dram3 & -0.12 & 0.02 & -5.76 & 0 \\
dram5 & -0.25 & 0.03 & -8.04 & 0 \\
dram6 & -0.17 & 0.03 & -5.47 & 0 \\
dram7 & -0.10 & 0.04 & -2.57 & 0.01 \\
dram8 & -0.20 & 0.04 & -5.24 & 0 \\
dram9 & 0.02 & 0.03 & 0.52 & 0.604 \\
dram10 & -0.09 & 0.03 & -2.83 & 0.005 \\
dram11 & 0.15 & 0.05 & 3.23 & 0.001 \\
dtam4 & 0.06 & 0.02 & 3.6 & 0 \\
dtam5 & 0.12 & 0.02 & 5.89 & 0 \\
dtam6 & 0.15 & 0.03 & 6 & 0 \\
dtam8 & -0.01 & 0.04 & -0.26 & 0.795 \\
docup1 & 0.37 & 0.04 & 8.9 & 0 \\
docup2 & 0.49 & 0.05 & 9.64 & 0 \\
docup3 & 0.92 & 0.08 & 11.56 & 0 \\
docup4 & 0.23 & 0.04 & 6.26 & 0 \\
docup5 & 0.20 & 0.02 & 11.38 & 0 \\
docup6 & 0.23 & 0.03 & 6.78 & 0 \\
docup7 & 0.28 & 7.35 & 0 \\
docup8 & 0.16 & 0.03 & 4.93 & 0 \\
docup9 & 0.04 & 1.8 & 0.071 \\
Constante & 0.04 & 71.03 & 0 \\
\hline
\end{tabular}


Cuadro A2(a). Complemento a los resultados de regresión del modelo Heckman aplicado a asalariados (primera parte) Variable dependiente: logaritmo natural del salario por hora

\begin{tabular}{|c|c|c|c|c|}
\hline Var dep: ling $x$ hrr & Coeficiente & Bootstrapp E.S & $Z$ & p-value \\
\hline \multicolumn{5}{|c|}{ En este lugar van las variables incluidas en el cuadro 2} \\
\hline ocmenos $35 \mathrm{~h}$ & 0.46 & 0.17 & 2.69 & 0.007 \\
\hline ocmas $35 \mathrm{hsm}$ & -1.11 & 0.06 & -17.29 & 0 \\
\hline ocmas $48 \mathrm{~h} 2 \mathrm{sm}$ & -0.45 & 0.04 & -12.46 & 0 \\
\hline ded_1 & 0.12 & 0.04 & 3.05 & 0.002 \\
\hline ded_2 & 0.11 & 0.06 & 2.07 & 0.038 \\
\hline ded_3 & 0.17 & 0.05 & 3.2 & 0.001 \\
\hline dcd_4 & 0.12 & 0.06 & 1.82 & 0.069 \\
\hline dcd_5 & 0.22 & 0.08 & 2.9 & 0.004 \\
\hline ded_7 & 0.15 & 0.10 & 1.5 & 0.132 \\
\hline dcd_8 & 0.07 & 0.07 & 0.97 & 0.332 \\
\hline dcd_9 & 0.15 & 0.06 & 2.57 & 0.01 \\
\hline dcd_10 & 0.02 & 0.10 & 0.2 & 0.84 \\
\hline dcd_12 & 0.19 & 0.10 & 1.89 & 0.058 \\
\hline dcd_13 & -0.14 & 0.08 & -1.74 & 0.081 \\
\hline dcd_14 & 0.11 & 0.06 & 1.8 & 0.072 \\
\hline dcd_15 & 0.12 & 0.09 & 1.29 & 0.198 \\
\hline dcd_16 & -0.02 & 0.11 & -0.21 & 0.831 \\
\hline dcd_17 & 0.01 & 0.18 & 0.03 & 0.975 \\
\hline dcd_18 & 0.31 & 0.07 & 4.23 & 0 \\
\hline dcd_19 & -0.11 & 0.08 & -1.45 & 0.148 \\
\hline dcd_21 & 0.13 & 0.08 & 1.64 & 0.102 \\
\hline dcd_24 & 0.14 & 0.09 & 1.54 & 0.124 \\
\hline dcd_25 & 0.16 & 0.09 & 1.83 & 0.068 \\
\hline dcd_26 & -0.13 & 0.09 & -1.48 & 0.139 \\
\hline dcd_27 & 0.24 & 0.08 & 2.87 & 0.004 \\
\hline dcd_28 & 0.06 & 0.08 & 0.73 & 0.466 \\
\hline dcd_29 & -0.17 & 0.11 & -1.58 & 0.114 \\
\hline dcd_31 & -0.04 & 0.10 & -0.41 & 0.684 \\
\hline dcd_32 & 0.17 & 0.11 & 1.52 & 0.13 \\
\hline dcd_33 & 0.27 & 0.12 & 2.29 & 0.022 \\
\hline ded_36 & 0.17 & 0.07 & 2.37 & 0.018 \\
\hline
\end{tabular}




\begin{tabular}{|lrrrr|} 
dcd_39 & 0.03 & 0.09 & 0.38 & 0.704 \\
dcd_40 & 0.26 & 0.09 & 3.04 & 0.002 \\
dcd_41 & 0.20 & 0.08 & 2.54 & 0.011 \\
dcd_43 & 0.02 & 0.08 & 0.27 & 0.784 \\
dcd_81 & 0.13 & 0.05 & 2.97 & 0.003 \\
dcd_82 & -0.10 & 0.05 & -2.18 & 0.029 \\
dcd_83 & -0.03 & 0.05 & -0.55 & 0.579 \\
dcd_84 & -0.01 & 0.04 & -0.17 & 0.864 \\
dcd85 & 0.01 & 0.06 & 0.24 & 0.813 \\
docup1 & 0.87 & 0.08 & 11.38 & 0 \\
docup2 & 0.69 & 0.22 & 3.07 & 0.002 \\
docup3 & 1.15 & 0.10 & 11.88 & 0 \\
docup4 & 1.01 & 0.30 & 3.41 & 0.001 \\
docup5 & 0.40 & 0.06 & 6.84 & 0 \\
docup6 & 0.49 & 0.07 & 7.31 & 0 \\
docup7 & 0.53 & 0.09 & 5.92 & 0 \\
docup8 & 0.56 & 0.07 & 7.75 & 0 \\
docup9 & 1.38 & 0.08 & 16.38 & 0 \\
Constante & 2.13 & 0.09 & 22.61 & 0 \\
\hline
\end{tabular}

Cuadro A2(b).Complemento a los resultados de regresión del modelo Heckman aplicado a asalariados (segunda parte)

Ecuación de selección:usapc

\begin{tabular}{|lrrrr|}
\hline Variables & Coeficiente & Bootstrapp E.S & \multicolumn{1}{c|}{$Z$} & \multicolumn{1}{c|}{$p$-value } \\
\hline esco & 0.23 & 0.01 & 23.02 & 0 \\
sexo & 0.03 & 0.04 & 0.63 & 0.529 \\
docup1 & 0.87 & 0.13 & 6.62 & 0 \\
docup2 & 0.79 & 0.18 & 4.44 & 0 \\
docup3 & 0.85 & 0.34 & 2.51 & 0.012 \\
docup4 & 1.11 & 0.08 & 13.98 & 0 \\
docup5 & 0.15 & 0.05 & 2.79 & 0.005 \\
docup6 & 0.64 & 0.07 & 8.91 & 0 \\
docup7 & -0.02 & 0.11 & -0.22 & 0.827 \\
Constante & -2.65 & 0.10 & -26.84 & 0 \\
/athrho & 0.85 & 0.10 & 8.56 & 0 \\
lnsigma & -0.68 & 0.03 & -20.18 & 0 \\
rho & 0.69 & 0.05 & & \\
sigma & 0.50 & 0.02 & & \\
lambda & 0.35 & 0.04 & & \\
\hline
\end{tabular}

LR test of indep. eqns. $($ rho $=0): \operatorname{chi} 2(1)=62.26$ Prob $>\operatorname{chi} 2=0.0001$ 


\section{Cuadro A3.}

Complemento a los resultados de regresión del modelo MCO aplicado a trabajadores por su cuenta..

Heckman aplicado a asalariados (primera parte)

\begin{tabular}{|c|c|c|c|c|}
\hline Var dep: ling $x$ hrr & Coeficiente & Bootstrapp E.S & $Z$ & p-value \\
\hline \multicolumn{5}{|c|}{ En este lugar van las variables incluidas en el cuadro 2} \\
\hline usapc & 0.18 & 0.05 & 3.42 & 0.001 \\
\hline esco & 0.02 & 0.01 & 2.86 & 0.004 \\
\hline sexo & 0.37 & 0.05 & 6.83 & 0 \\
\hline edocivil & -0.02 & 0.05 & -0.32 & 0.752 \\
\hline experi & 0.02 & 0.01 & 3.51 & 0 \\
\hline experi2 & 0.00 & 0.00 & -2.97 & 0.003 \\
\hline hrsocup & -0.01 & 0.00 & -12.51 & 0 \\
\hline ocmenos $35 \mathrm{~h}$ & 0.43 & 0.08 & 5.17 & 0 \\
\hline ocmas $35 \mathrm{hsm}$ & -1.24 & 0.04 & -28.10 & 0 \\
\hline ocmas $48 \mathrm{~h} 2 \mathrm{sm}$ & -0.44 & 0.05 & -8.87 & 0 \\
\hline dcd_1 & 0.33 & 0.12 & 2.79 & 0.005 \\
\hline dcd_2 & 0.48 & 0.21 & 2.34 & 0.019 \\
\hline dcd_3 & 0.29 & 0.13 & 2.17 & 0.03 \\
\hline dcd_4 & 0.38 & 0.17 & 2.32 & 0.02 \\
\hline dcd_5 & 0.31 & 0.14 & 2.19 & 0.028 \\
\hline dcd_7 & 0.40 & 0.11 & 3.56 & 0 \\
\hline dcd_8 & -0.01 & 0.17 & -0.03 & 0.976 \\
\hline dcd_9 & 0.59 & 0.47 & 1.25 & 0.21 \\
\hline ded_10 & 0.15 & 0.19 & 0.79 & 0.428 \\
\hline ded_12 & 0.31 & 0.09 & 3.65 & 0 \\
\hline dcd_13 & 0.27 & 0.14 & 1.96 & 0.05 \\
\hline $\mathrm{dcd}=14$ & 0.50 & 0.13 & 3.90 & 0 \\
\hline dcd_15 & 0.71 & 0.22 & 3.18 & 0.001 \\
\hline ded_16 & 0.55 & 0.19 & 2.88 & 0.004 \\
\hline dcd_17 & 0.74 & 0.40 & 1.87 & 0.062 \\
\hline dcd_18 & 0.07 & 0.23 & 0.33 & 0.741 \\
\hline ded_19 & 0.14 & 0.21 & 0.67 & 0.504 \\
\hline dcd_21 & 0.37 & 0.25 & 1.50 & 0.135 \\
\hline dcd_24 & 0.42 & 0.15 & 2.77 & 0.006 \\
\hline ded_25 & 1.34 & 0.37 & 3.68 & 0 \\
\hline ded_26 & 0.32 & 0.23 & 1.37 & 0.171 \\
\hline ded_27 & 0.38 & 0.11 & 3.58 & 0 \\
\hline dcd_28 & 0.28 & 0.14 & 2.06 & 0.039 \\
\hline ded_29 & 0.05 & 0.14 & 0.37 & 0.712 \\
\hline dcd_31 & 0.35 & 0.15 & 2.30 & 0.022 \\
\hline dcd_32 & 0.67 & 0.07 & 9.22 & 0 \\
\hline ded_33 & 0.50 & 0.17 & 2.98 & 0.003 \\
\hline dcd_36 & 0.40 & 0.16 & 2.56 & 0.011 \\
\hline dcd_39 & 0.14 & 0.11 & 1.24 & 0.215 \\
\hline ded_40 & 0.58 & 0.10 & 5.58 & 0 \\
\hline
\end{tabular}




\begin{tabular}{|llrrr|} 
dcd_41 & 0.18 & 0.12 & 1.53 & 0.125 \\
dcd_43 & 0.24 & 0.14 & 1.72 & 0.085 \\
dcd_81 & 0.31 & 0.06 & 5.59 & 0 \\
dcd_82 & 0.24 & 0.08 & 3.01 & 0.003 \\
dcd_83 & 0.23 & 0.06 & 4.05 & 0 \\
dcd_84 & 0.25 & 0.07 & 3.39 & 0.001 \\
dcd_85 & 0.25 & 0.08 & 3.23 & 0.001 \\
docup1 & 0.87 & 0.08 & 11.38 & 0 \\
docup2 & 0.69 & 0.22 & 3.07 & 0.002 \\
docup3 & 1.15 & 0.10 & 11.88 & 0 \\
docup4 & 1.01 & 0.30 & 3.41 & 0.001 \\
docup5 & 0.40 & 0.06 & 6.84 & 0 \\
docup6 & 0.49 & 0.07 & 7.31 & 0 \\
docup7 & 0.53 & 0.09 & 5.92 & 0 \\
docup8 & 0.56 & 0.07 & 7.75 & 0 \\
docup9 & 1.38 & 0.08 & 16.38 & 0 \\
Constante & 2.13 & 0.09 & 22.61 & 0 \\
\hline
\end{tabular}


